\title{
EXISTENCE AND COMPARISON RESULTS FOR VARIATIONAL-HEMIVARIATIONAL INEQUALITIES
}

\author{
S. CARL
}

Received 18 June 2004

We consider a prototype of quasilinear elliptic variational-hemivariational inequalities involving the indicator function of some closed convex set and a locally Lipschitz functional. We provide a generalization of the fundamental notion of sub- and supersolutions on the basis of which we then develop the sub-supersolution method for variationalhemivariational inequalities. Furthermore, we give an example to illustrate the abstract theory developed in this paper.

\section{Introduction}

Let $\Omega \subset \mathbb{R}^{N}$ be a bounded domain with Lipschitz boundary $\partial \Omega$, and let $V=W^{1, p}(\Omega)$ and $V_{0}=W_{0}^{1, p}(\Omega), 1<p<\infty$, denote the usual Sobolev spaces with their dual spaces $V^{*}$ and $V_{0}^{*}$, respectively. In this paper, we deal with the following variational-hemivariational inequality:

$$
u \in K:\left\langle-\Delta_{p} u-f, v-u\right\rangle+\int_{\Omega} j^{o}(u ; v-u) d x \geq 0, \quad \forall v \in K
$$

where $j^{o}(s ; r)$ denotes the generalized directional derivative of the locally Lipschitz function $j: \mathbb{R} \rightarrow \mathbb{R}$ at $s$ in the direction $r$ given by

$$
j^{o}(s ; r)=\limsup _{y \rightarrow s, t \downarrow 0} \frac{j(y+t r)-j(y)}{t},
$$

(cf., e.g., [3, Chapter 2]), and $K \subset V_{0}$ is some closed and convex subset. The operator $\Delta_{p} u=\operatorname{div}\left(|\nabla u|^{p-2} \nabla u\right)$ is the $p$-Laplacian, $1<p<\infty$, and $f \in V_{0}^{*}$.

The main goal of this paper is to develop the sub-supersolution method for variationalhemivariational inequalities of the form (1.1) which may be considered as a prototype of more general problems of this kind. Problem (1.1) includes various special cases: 
(i) for $K=V_{0}$ and $j: \mathbb{R} \rightarrow \mathbb{R}$ smooth, (1.1) is the weak formulation of the Dirichlet problem

$$
u \in V_{0}:-\Delta_{p} u+j^{\prime}(u)=f \text { in } V_{0}^{*},
$$

for which the sub-supersolution method is well known,

(ii) if $K=V_{0}$, and $j: \mathbb{R} \rightarrow \mathbb{R}$ not necessarily smooth, then (1.1) is a hemivariational inequality of the form

$$
u \in V_{0}:\left\langle-\Delta_{p} u-f, v-u\right\rangle+\int_{\Omega} j^{o}(u ; v-u) d x \geq 0, \quad \forall v \in V_{0},
$$

for which an extension of the sub-supersolution method has been given recently in $[2]$,

(iii) if $j=0$, then (1.1) becomes a variational inequality for which a sub-supersolution method has been developed in $[4,5]$, and an extension of this method to systems of variational inequalities has been proved recently in [1].

This paper provides a unified theory on the sub-supersolution method for variationalhemivariational inequalities that includes all the above cited special cases.

\section{Notation and hypotheses}

For functions $w, z: \Omega \rightarrow \mathbb{R}$ and sets $W$ and $Z$ of functions defined on $\Omega$, we use the notations: $w \wedge z=\min \{w, z\}, w \vee z=\max \{w, z\}, W \wedge Z=\{w \wedge z \mid w \in W, z \in Z\}$, $W \vee Z=\{w \vee z \mid w \in W, z \in Z\}$, and $w \wedge Z=\{w\} \wedge Z, w \vee Z=\{w\} \vee Z$. Next we introduce our basic notion of sub-supersolution.

Definition 2.1. A function $\underline{u} \in V$ is called a subsolution of (1.1) if the following holds:

(i) $\underline{u} \leq 0$ on $\partial \Omega$,

(ii) $\left\langle-\Delta_{p} \underline{u}-f, v-\underline{u}\right\rangle+\int_{\Omega} j^{o}(\underline{u} ; v-\underline{u}) d x \geq 0, \forall v \in \underline{u} \wedge K$.

Definition 2.2. $\bar{u} \in V$ is a supersolution of (1.1) if the following holds:

(i) $\bar{u} \geq 0$ on $\partial \Omega$,

(ii) $\left\langle-\Delta_{p} \bar{u}-f, v-\bar{u}\right\rangle+\int_{\Omega} j^{o}(\bar{u} ; v-\bar{u}) d x \geq 0, \forall v \in \bar{u} \vee K$.

Let $\partial j: \mathbb{R} \rightarrow 2^{\mathbb{R}} \backslash\{\varnothing\}$ denote Clarke's generalized gradient of $j$ defined by

$$
\partial j(s):=\left\{\zeta \in \mathbb{R} \mid j^{o}(s ; r) \geq \zeta r, \forall r \in \mathbb{R}\right\}
$$

We assume the following hypothesis for $j$.

(H) The function $j: \mathbb{R} \rightarrow \mathbb{R}$ is locally Lipschitz and its Clarke's generalized gradient $\partial j$ satisfies the following growth conditions:

(i) there exists a constant $c_{1} \geq 0$ such that

$$
\xi_{1} \leq \xi_{2}+c_{1}\left(s_{2}-s_{1}\right)^{p-1}
$$

for all $\xi_{i} \in \partial j\left(s_{i}\right), i=1,2$, and for all $s_{1}, s_{2}$ with $s_{1}<s_{2}$, 
(ii) there is a constant $c_{2} \geq 0$ such that

$$
\xi \in \partial j(s):|\xi| \leq c_{2}\left(1+|s|^{p-1}\right), \quad \forall s \in \mathbb{R} .
$$

Let $L^{p}(\Omega)$ be equipped with the natural partial ordering of functions defined by $u \leq w$ if and only if $w-u$ belongs to the positive cone $L_{+}^{p}(\Omega)$ of all nonnegative elements of $L^{p}(\Omega)$. This induces a corresponding partial ordering also in the subspace $V$ of $L^{p}(\Omega)$, and if $u, w \in V$ with $u \leq w$ then

$$
[u, w]=\{z \in V \mid u \leq z \leq w\}
$$

denotes the order interval formed by $u$ and $w$.

In the proofs of our main results we make use of the cut-off function $b: \Omega \times \mathbb{R} \rightarrow \mathbb{R}$ related with an ordered pair of functions $\underline{\mathcal{u}} \bar{u}$, and given by

$$
b(x, s)= \begin{cases}(s-\bar{u}(x))^{p-1} & \text { if } s>\bar{u}(x), \\ 0 & \text { if } \underline{u}(x) \leq s \leq \bar{u}(x), \\ -(\underline{u}(x)-s)^{p-1} & \text { if } s<\underline{u}(x) .\end{cases}
$$

One readily verifies that $b$ is a Carathéodory function satisfying the growth condition

$$
|b(x, s)| \leq k(x)+c_{3}|s|^{p-1}
$$

for a.e. $x \in \Omega$, for all $s \in \mathbb{R}$, with some function $k \in L_{+}^{q}(\Omega)$, and $q$ satisfying $1 / p+1 / q=1$. Moreover, one has the following estimate

$$
\int_{\Omega} b(x, u(x)) u(x) d x \geq c_{4}\|u\|_{L^{p}(\Omega)}^{p}-c_{5}, \quad \forall u \in L^{p}(\Omega),
$$

where $c_{4}$ and $c_{5}$ are some positive constants. In view of (2.6), the Nemytskij operator $B: L^{p}(\Omega) \rightarrow L^{q}(\Omega)$ defined by

$$
B u(x)=b(x, u(x))
$$

is continuous and bounded, and thus due to the compact embedding $V \subset L^{p}(\Omega)$ it follows that $B: V_{0} \rightarrow V_{0}^{*}$ is compact.

\section{Preliminaries}

In this section, we briefly recall a surjectivity result for multivalued mappings in reflexive Banach spaces (cf., e.g., [6, Theorem 2.12]) which among others will be used in the proof of our main result.

Theorem 3.1. Let $X$ be a real reflexive Banach space with dual space $X^{*}, \Phi: X \rightarrow 2^{X^{*}}$ a maximal monotone operator, and $u_{0} \in \operatorname{dom}(\Phi)$. Let $A: X \rightarrow 2^{X^{*}}$ be a pseudomonotone operator, and assume that either $A_{u_{0}}$ is quasi-bounded or $\Phi_{u_{0}}$ is strongly quasi-bounded. Assume further that $A: X \rightarrow 2^{X^{*}}$ is $u_{0}$-coercive, that is, there exists a real-valued function $c: \mathbb{R}_{+} \rightarrow \mathbb{R}$ with $c(r) \rightarrow+\infty$ as $r \rightarrow+\infty$ such that for all $\left(u, u^{*}\right) \in \operatorname{graph}(A)$ one has $\left\langle u^{*}\right.$, $\left.u-u_{0}\right\rangle \geq c\left(\|u\|_{X}\right)\|u\|_{X}$. Then $A+\Phi$ is surjective, that is, range $(A+\Phi)=X^{*}$. 
The operators $A_{u_{0}}$ and $\Phi_{u_{0}}$ that appear in the theorem above are defined by $A_{u_{0}}(v):=$ $A\left(u_{0}+v\right)$ and similarly for $\Phi_{u_{0}}$. As for the notion of quasi-bounded and strongly quasibounded, we refer to [6, page 51]. In particular, one has that any bounded operator is quasi-bounded and strongly quasi-bounded as well. The following proposition provides sufficient conditions for an operator $A: X \rightarrow 2^{X^{*}}$ to be pseudomonotone, which is suitable for our purpose.

Proposition 3.2. Let $X$ be a real reflexive Banach space, and assume that $A: X \rightarrow 2^{X^{*}}$ satisfies the following conditions:

(i) for each $u \in X$ we have that $A(u)$ is a nonempty, closed and convex subset of $X^{*}$;

(ii) $A: X \rightarrow 2^{X^{*}}$ is bounded;

(iii) if $u_{n} \rightarrow u$ in $X$ and $u_{n}^{*} \rightarrow u^{*}$ in $X^{*}$ with $u_{n}^{*} \in A\left(u_{n}\right)$ and if $\lim \sup \left\langle u_{n}^{*}, u_{n}-u\right\rangle \leq 0$, then $u^{*} \in A(u)$ and $\left\langle u_{n}^{*}, u_{n}\right\rangle \rightarrow\left\langle u^{*}, u\right\rangle$.

Then the operator $A: X \rightarrow 2^{X^{*}}$ is pseudomonotone.

As for the proof of Proposition 3.2 we refer, for example, to [6, Chapter 2].

\section{Main result}

The main result of this paper is given by the following theorem which provides an existence and comparison result for the variational-hemivariational inequality (1.1).

THeOREM 4.1. Let $\bar{u}$ and $\underline{u}$ be super- and subsolutions of (1.1), respectively, satisfying $\underline{u} \leq \bar{u}$, and assume $\bar{u} \wedge K \subset K$ and $\underline{u} \vee K \subset K$. Then under hypothesis $(H)$ there exist solutions of (1.1) within the order interval $[\underline{u}, \bar{u}]$.

Proof. Let $I_{K}: V_{0} \rightarrow \mathbb{R} \cup\{+\infty\}$ denote the indicator function related with the given closed convex set $K \neq \varnothing$ and defined by

$$
I_{K}(u)= \begin{cases}0 & \text { if } u \in K, \\ +\infty & \text { if } u \notin K,\end{cases}
$$

which is proper, convex, and lower semicontinuous. By means of the indicator function the variational-hemivariational inequality (1.1) can be rewritten in the following form. Find $u \in V_{0}$ such that

$$
\left\langle-\Delta_{p} u-f, v-u\right\rangle+I_{K}(v)-I_{K}(u)+\int_{\Omega} j^{o}(u ; v-u) d x \geq 0, \quad \forall v \in V_{0} .
$$

Since we are looking for solutions of (4.2) within $[\underline{u}, \bar{u}]$, we consider the following auxiliary problem. Find $u \in V_{0}$ such that

$$
\left\langle-\Delta_{p} u-f+\lambda B(u), v-u\right\rangle+I_{K}(v)-I_{K}(u)+\int_{\Omega} j^{o}(u ; v-u) d x \geq 0, \quad \forall v \in V_{0},
$$

where $B$ is the cut-off operator introduced in Section 2, and $\lambda \geq 0$ is some parameter to be specified later. As will be seen in the course of the proof, the role of $\lambda B$ is twofold. First it provides a coercivity generating term, and second, it allows for comparison. The proof of the theorem will be done in two steps. In Step 1, we prove the existence of solutions of 
the auxiliary problem (4.3), and in Step 2, we are going to show that any solution of (4.3) belongs to the interval $[\underline{u}, \bar{u}]$, which completes the proof, since then $B(u)=0$ and $(4.2)$ holds.

Step 1 (existence for (4.3)). We introduce the functional $J: L^{p}(\Omega) \rightarrow \mathbb{R}$ defined by

$$
J(v)=\int_{\Omega} j(v(x)) d x, \quad \forall v \in L^{p}(\Omega),
$$

which by hypothesis $(\mathrm{H})$ is locally Lipschitz, and moreover, by Aubin-Clarke theorem (see [3, page 83]) for each $u \in L^{p}(\Omega)$ we have

$$
\xi \in \partial J(u) \Longrightarrow \xi \in L^{q}(\Omega) \text { with } \xi(x) \in \partial j(u(x)) \text { for a.e. } x \in \Omega \text {. }
$$

Consider now the multivalued operator

$$
-\Delta_{p}+\lambda B+\partial\left(\left.J\right|_{V_{0}}\right)+\partial I_{K}: V_{0} \longrightarrow 2^{V_{0}^{*}}
$$

where $\left.J\right|_{V_{0}}$ denotes the restriction of $J$ to $V_{0}$ and $\partial I_{K}$ is the subdifferential of $I_{K}$ in the sense of convex analysis. It is well known that $\Phi:=\partial I_{K}: V_{0} \rightarrow 2^{V_{0}^{*}}$ is a maximal monotone operator, (cf., e.g., [7]). Since $-\Delta_{p}: V_{0} \rightarrow V_{0}^{*}$ is strictly monotone, bounded, and continuous, and $\lambda B: V_{0} \rightarrow V_{0}^{*}$ is bounded, continuous and compact, it follows that $-\Delta_{p}+\lambda B: V_{0} \rightarrow$ $V_{0}^{*}$ is a (singlevalued) pseudomonotone, continuous, and bounded operator. In [2], it has been shown that $\partial\left(\left.J\right|_{V_{0}}\right): V_{0} \rightarrow 2^{V_{0}^{*}}$ is a (multivalued) pseudomonotone operator, which, due to $(\mathrm{H})$, is bounded. Thus $A:=-\Delta_{p}+\lambda B+\partial\left(\left.J\right|_{V_{0}}\right): V_{0} \rightarrow 2^{V_{0}^{*}}$ is a pseudomonotone and bounded operator. Hence, it follows by Theorem 3.1 that $\operatorname{range}(A+\Phi)=V_{0}^{*}$ provided $A$ is $u_{0}$-coercive for some $u_{0} \in K$, which can readily be seen as follows: for any $v \in V_{0}$ and any $w \in \partial\left(\left.J\right|_{V_{0}}\right)(v)$, we obtain by applying $(\mathrm{H})(\mathrm{ii})$ and (2.7) the estimate

$$
\begin{aligned}
& \left\langle-\Delta_{p} v+\lambda B(v)+w, v-u_{0}\right\rangle \\
& \quad=\int_{\Omega}|\nabla v|^{p} d x+\lambda\langle B(v), v\rangle+\int_{\Omega} w v d x-\left\langle-\Delta_{p} v+\lambda B(v)+w, u_{0}\right\rangle \\
& \quad \geq \int_{\Omega}|\nabla v|^{p} d x+c_{4} \lambda\|v\|_{L^{p}(\Omega)}^{p}-c_{5} \lambda-c_{2} \int_{\Omega}\left(1+|v|^{p-1}\right)|v| d x \\
& \quad-\left|\left\langle-\Delta_{p} v+\lambda B(v)+w, u_{0}\right\rangle\right| \geq\|v\|_{V_{0}}^{p}-C\left(1+\|v\|_{V_{0}}^{p-1}\right),
\end{aligned}
$$

for some constant $C>0$, by choosing the constant $\lambda$ in such a way that $c_{4} \lambda>c_{2}$. Since $p>1$, the coercivity of $A$ follows from (4.7). In view of the surjectivity of the operator $A+\Phi$ there exists a $u \in \operatorname{dom}(A) \cap \operatorname{dom}(\Phi)$ such that $f \in A(u)+\Phi(u)$, that is, there is an $\xi \in \partial\left(\left.J\right|_{V_{0}}\right)(u)$ with $\xi \in L^{q}(\Omega)$ and $\xi(x) \in \partial j(u(x))$ for a.e. $x \in \Omega$, and an $\eta \in \Phi(u)$ such that

$$
-\Delta_{p} u-f+\lambda B(u)+\xi+\eta=0 \quad \text { in } V_{0}^{*}
$$

where

$$
\begin{aligned}
& \langle\xi, \varphi\rangle=\int_{\Omega} \xi(x) \varphi(x) d x, \quad \forall \varphi \in V_{0}, \\
& I_{K}(v) \geq I_{K}(u)+\langle\eta, v-u\rangle, \quad \forall v \in V_{0} .
\end{aligned}
$$


By the definition of Clarke's generalized gradient $\partial j$ from (4.9) we get

$$
\langle\xi, \varphi\rangle=\int_{\Omega} \xi(x) \varphi(x) d x \leq \int_{\Omega} j^{o}(u(x) ; \varphi(x)) d x, \quad \forall \varphi \in V_{0} .
$$

Thus from (4.8), (4.9), (4.10), and (4.11) with $\varphi$ replaced by $v-u$, we obtain (4.3), which proves the existence of solutions of problem (4.3).

Step 2 ( $\underline{u} \leq u \leq \bar{u}$ for any solution $u$ of (4.3)). We first show $u \leq \bar{u}$. By definition, the supersolution $\bar{u}$ satisfies: $\bar{u} \geq 0$ on $\partial \Omega$, and

$$
\left\langle-\Delta_{p} \bar{u}-f, v-\bar{u}\right\rangle+\int_{\Omega} j^{o}(\bar{u} ; v-\bar{u}) d x \geq 0, \quad \forall v \in \bar{u} \vee K .
$$

Let $u$ be any solution of (4.3) which is equivalent with the following variationalhemivariational inequality:

$$
u \in K:\left\langle-\Delta_{p} u-f, v-u\right\rangle+\langle\lambda B(u), v-u\rangle+\int_{\Omega} j^{o}(u ; v-u) d x \geq 0, \quad \forall v \in K .
$$

We apply the special test function $v=\bar{u} \vee u=\bar{u}+(u-\bar{u})^{+}$in (4.12) and $v=\bar{u} \wedge u=$ $u-(u-\bar{u})^{+}$in $(4.13)$, and get by adding the resulting inequalities the following one:

$$
\begin{aligned}
& \left\langle-\Delta_{p} \bar{u}-\left(-\Delta_{p} u\right),(u-\bar{u})^{+}\right\rangle+\lambda\left\langle B(u),-(u-\bar{u})^{+}\right\rangle \\
& \quad+\int_{\Omega}\left(j^{o}\left(\bar{u} ;(u-\bar{u})^{+}\right)+j^{o}\left(u ;-(u-\bar{u})^{+}\right)\right) d x \geq 0,
\end{aligned}
$$

which yields, due to

$$
\left\langle-\Delta_{p} u-\left(-\Delta_{p} \bar{u}\right),(u-\bar{u})^{+}\right\rangle \geq 0,
$$

the inequality

$$
\lambda\left\langle B(u),(u-\bar{u})^{+}\right\rangle \leq \int_{\Omega}\left(j^{o}\left(\bar{u} ;(u-\bar{u})^{+}\right)+j^{o}\left(u ;-(u-\bar{u})^{+}\right)\right) d x .
$$

By using $(\mathrm{H})$ and the properties on $j^{o}$ and $\partial j$, we get for certain $\bar{\xi}(x) \in \partial j(\bar{u}(x))$ and $\xi(x) \in \partial j(u(x))$ the following estimate of the right-hand side of (4.16):

$$
\begin{aligned}
& \int_{\Omega}\left(j^{o}\left(\bar{u} ;(u-\bar{u})^{+}\right)+j^{o}\left(u ;-(u-\bar{u})^{+}\right)\right) d x \\
& \quad=\int_{\{u>\bar{u}\}}\left(j^{o}(\bar{u} ; u-\bar{u})+j^{o}(u ;-(u-\bar{u}))\right) d x \\
& \quad=\int_{\{u>\bar{u}\}}(\bar{\xi}(x)(u(x)-\bar{u}(x))+\xi(x)(-(u(x)-\bar{u}(x)))) d x \\
& \quad=\int_{\{u>\bar{u}\}}(\bar{\xi}(x)-\xi(x))(u(x)-\bar{u}(x)) d x \\
& \quad \leq \int_{\{u>\bar{u}\}} c_{1}(u(x)-\bar{u}(x))^{p} d x .
\end{aligned}
$$


Since

$$
\left\langle B(u),(u-\bar{u})^{+}\right\rangle=\int_{\{u>\bar{u}\}}(u-\bar{u})^{p} d x,
$$

we get from (4.16) and (4.17) the estimate

$$
\left(\lambda-c_{1}\right) \int_{\{u>\bar{u}\}}(u-\bar{u})^{p} d x \leq 0 .
$$

Selecting the parameter $\lambda$, in addition, such that $\lambda-c_{1}>0$ then (4.19) yields

$$
\int_{\Omega}\left((u-\bar{u})^{+}\right)^{p} d x \leq 0
$$

which implies $(u-\bar{u})^{+}=0$ and thus $u \leq \bar{u}$. The proof for the inequality $\underline{u} \leq u$ can be carried out in a similar way which completes the proof of the theorem.

4.1. Example. We consider (1.1) with $f \in L^{\infty}(\Omega)$ and $K$ representing the following obstacle problem

$$
K=\left\{v \in V_{0} \mid v(x) \leq \psi(x) \text { for a.e. } x \in \Omega\right\},
$$

and assume $\psi \in L^{\infty}(\Omega)$. We are going to provide sufficient conditions for the existence of an ordered pair of constant sub- and supersolutions $\alpha$ and $\beta$, respectively.

Proposition 4.2. Let $K$ be given by (4.21) and assume $f, \psi \in L^{\infty}(\Omega)$. Then the following hold.

(a) the constant function $\underline{u}(x) \equiv \alpha \leq 0$ is a subsolution of (1.1) if

$$
f(x) \geq-j^{o}(\alpha ;-1) \quad \text { for a.e. } x \in \Omega \text {; }
$$

(b) the constant function $\bar{u}(x) \equiv \beta \geq 0$ is a supersolution of (1.1) if

$$
f(x) \leq j^{o}(\beta ; 1) \text { for a.e. } x \in \Omega \text {. }
$$

Proof. Let $\alpha \leq 0$ satisfy (4.22). According to Definition 2.1, we only need to verify that $\alpha$ satisfies (ii) in Definition 2.1. To this end let $v \in \alpha \wedge K$ be given. Then $v-\alpha \leq 0$ in $\Omega$ and in view of (4.22) we get

$$
\begin{aligned}
& \left\langle-\Delta_{p} \alpha-f, v-\alpha\right\rangle+\int_{\Omega} j^{o}(\alpha ; v(x)-\alpha) d x \\
& \quad=\int_{\Omega}\left(j^{o}(\alpha ; v(x)-\alpha)-f(x)(v(x)-\alpha)\right) d x \\
& =\int_{\Omega}\left(j^{o}(\alpha ;-1)+f(x)\right)(\alpha-v(x)) d x \geq 0 \quad \forall v \in \alpha \wedge K,
\end{aligned}
$$

which proves that $\alpha$ is a subsolution. In a similar way one can show that under (4.23) the constant $\beta \geq 0$ is a supersolution. 
In order to apply Theorem 4.1 to our example, we only need to make sure that, in addition, $\beta \wedge K \subset K$ and $\alpha \vee K \subset K$ is satisfied. For the obstacle problem $\beta \wedge K \subset K$ is trivially satisfied and $\alpha \vee K \subset K$ holds provided $\alpha \leq \psi(x)$ for a.e. $x \in \Omega$.

4.2. Remarks. (i) As already mentioned problem (1.1) can be treated also with the $p$ Laplacian replaced by a more general quasilinear elliptic operator of Leray-Lions type involving lower order terms.

(ii) It can be shown that the set $\mathscr{S}$ of all solutions of (1.1) lying within the interval $[\underline{u}, \bar{u}]$ is a compact set in $V_{0}$.

(iii) Under additional lattice conditions such as $K \wedge K \subset K$ and $K \vee K \subset K$ one can prove that the solution set $\mathscr{S}$ possesses extremal elements, that is, there exist the greatest and smallest solution with respect to the underlying partial ordering.

\section{References}

[1] S. Carl and V. K. Le, Enclosure results for quasilinear systems of variational inequalities, J. Differential Equations 199 (2004), no. 1, 77-95.

[2] S. Carl, V. K. Le, and D. Motreanu, The sub-supersolution method and extremal solutions for quasilinear hemivariational inequalities, Differential Integral Equations 17 (2004), no. 1-2, $165-178$.

[3] F. H. Clarke, Optimization and Nonsmooth Analysis, Classics in Applied Mathematics, vol. 5, Society for Industrial and Applied Mathematics (SIAM), Pennsylvania, 1990.

[4] V. K. Le, Sub-supersolutions and the existence of extremal solutions in noncoercive variational inequalities, JIPAM. J. Inequal. Pure Appl. Math. 2 (2001), no. 2, Article 20, 16 pp.

[5] Subsolution-supersolution method in variational inequalities, Nonlinear Anal. Ser. A: Theory Methods 45 (2001), no. 6, 775-800.

[6] Z. Naniewicz and P. D. Panagiotopoulos, Mathematical Theory of Hemivariational Inequalities and Applications, Monographs and Textbooks in Pure and Applied Mathematics, vol. 188, Marcel Dekker, New York, 1995.

[7] E. Zeidler, Nonlinear Functional Analysis and Its Applications. II/B, Springer-Verlag, New York, 1990.

S. Carl: Fachbereich Mathematik und Informatik, Institut für Analysis, Martin-Luther-Universität Halle-Wittenberg, 06099 Halle, Germany

E-mail address: carl@mathematik.uni-halle.de 